\title{
Inversiones en recursos de uso y propiedad común para un desarrollo inclusivo y sostenible Lecciones de Guatemala, México, Nepal y Namibia
}

\author{
Sophia Gnych', Steven Lawry', Rebecca McLain', Iliana Monterroso', Anukram Adhikary²
}

\section{Mensajes claves}

- La inversión en bienes comunes proviene mayormente de donantes, gobiernos y comunidades, pero la inversión desde el sector privado está aumentando.

- La preparación para la inversión en bienes comunes depende en buena medida del nivel de confianza que tienen los actores interesados en que las obligaciones de cada parte serán cumplidas. La preparación para la inversión se desarrolla en el tiempo y en etapas, a medida que los niveles de confianza se incrementan.

- Cada sector tiene roles y responsabilidades específicas para mitigar el riesgo.

- Los derechos comunitarios promueven una inversión que reconoce el carácter social de la propiedad y del uso de bienes comunes y proporciona beneficios ambientales y sociales, así como ganancias.

- Existe la necesidad de mayor investigación sobre los orígenes, mecanismos, volumen y dirección de la inversión en recursos manejados por las comunidades que pueda ayudar a que las comunidades e inversionistas comprendan mejor sus alternativas.

\section{Introducción}

Las comunidades locales y los pueblos indígenas administran una porción significativa de los bosques remanentes, las tierras de pastoreo, y la pesca en el mundo como recursos de propiedad común (Wily, 2018). Transferir la propiedad, el uso y los derechos de exclusión a las comunidades puede, bajo muchas circunstancias, brindar incentivos para que ellas administren los recursos en formas que faciliten la gestión sostenible, así como mayor equidad en la distribución de beneficios (Baynes et al. 2015). Sin embargo, nuestro conocimiento sobre quién está invirtiendo en recursos de propiedad de las comunidades después de la devolución de derechos y cómo evolucionan los

Centro para la Investigación Forestal Internacional

2 Forest Action, Nepal patrones de inversión en el tiempo es fragmentado. Abordamos esta brecha en el conocimiento con la exploración de los mecanismos que han surgido para realizar inversiones en recursos de propiedad y uso común en Guatemala, México, Nepal y Namibia.

\section{Antecedentes}

Cuando se da un proceso de devolución de derechos, las comunidades como un todo son reconocidas como las titulares de los mismos y se empoderan las instituciones nuevas o existentes para gobernar los bienes comunes (Cronkleton et al. 2011; AmbroseOji et al. 2015). Cuando el proceso de devolución es sobre los derechos a los bosques, por ejemplo, surgen instituciones forestales a nivel comunal (IFC) que normalmente están compuestas por grupos de usuarios de la comunidad que desempeñan funciones de gobernanza forestal en conjunto con una o más empresas forestales comunitarias (EFC) diseñadas para captar el valor monetario de los bienes forestales comunes (Bray y Merino, 2002). Las IFC operan como empresas sociales, que son diferentes de las empresas con fines de lucro en la medida que las ganancias son "retenidas en sus organizaciones y/o comunidad tanto como servicios directos o subvenciones económicas para la población objetivo" (Foundjem-Tita et al. 2019, 5). Las EFC son vistas como un tipo de innovación social (Kluvánková et al. 2018), que es definida como "... la reconfiguración de prácticas sociales, en respuesta a desafíos societales, que busca mejorar los resultados sobre el bienestar social y necesariamente incluye el involucramiento de los actores de la sociedad civil" (Polman et al. 2017, 12).

Con frecuencia, la falta de capital financiero limita el crecimiento y éxito de las IFC. El concepto de preparación para las inversiones ha surgido como un instrumento para orientar las políticas y reducir las barreras para las inversiones en iniciativas (Mason y Kwok, 2010). Aquí se entiende la preparación para las inversiones como la capacidad de los administradores de las IFC para estar al tanto de las necesidades de los inversionistas, para abordarlos y proporcionarles información suficiente, así como para desarrollar credibilidad y confianza de manera que estos proporcionen financiamiento (Fellnhofer, 2015). 
La creación de IFC preparadas para la inversión requiere reducir los riesgos y los costos de transacción. Cuatro condiciones claves pueden disminuir los riesgos y brindar las garantías que los inversionistas necesitan para invertir en IFC: 1) derechos claros, seguros y suficientemente amplios (Lawry et al. 2017); 2) relaciones de confianza y redes sociales sólidas dentro de las comunidades y entre las comunidades y los actores externos (Baynes et al. 2015); 3) procedimientos y reglas claras y aplicables que rijan los bosques y empresas asociadas (Dasgupta, 2005) y 4) suficientes capacidades tecnológicas, de negociación y gestión dentro de la comunidad (Hewitt y Castro Delgadillo, 2009).

Facilitar las inversiones, con políticas que aclaran los derechos y alientan la transparencia y la responsabilidad, así como con programas que crean capacidades de monitoreo, ejecución, administrativas y técnicas, puede reducir los riesgos de forma suficiente para atraer el financiamiento externo para inversiones en

"Las inversiones que facilitan condiciones crean bienes públicos, los que a su vez permiten las inversiones en activos para crear activos privados. Estos activos privados...son los bienes creados por los propios titulares de los derechos: en empresas, ahorros privados, infraestructura física y mejor salud y educación" (Elson, 2012, 27). activos (Elson, 2012). Las organizaciones comunitarias a nivel local pueden formar asociaciones para aumentar su eficacia en configurar el contexto regulatorio, así como para reducir los riesgos y respaldar el financiamiento para las comunidades (Paudel et al. 2012).

Según los conceptos abordados anteriormente, hemos elaborado una teoría de cambio (TC) tentativa (Figura 1) para orientar nuestro estudio sobre las inversiones que ocurren después de procesos de devolución de derechos. Nuestra TC plantea un camino por el cual la devolución de derechos a las comunidades conlleva a inversiones y resulta en impactos sociales y medioambientales. En este estudio se busca desentrañar los mecanismos desconocidos de las inversiones descritas en el diagrama TC.

Planteamos nuestro análisis en torno a las siguientes premisas:

- Premisa 1: Las barreras a las inversiones en las IFC no son insalvables y tales inversiones se producen después de la devolución de derechos.

- Premisa 2: La preparación para las inversiones en las IFC requiere que los inversionistas potenciales y las empresas que reciben la inversión tengan garantía de que las obligaciones de cada parte serán cumplidas. Cada sector (por ejemplo, público, sociedad civil, privado) asume funciones y responsabilidades específicas para mitigar el riesgo.

\begin{tabular}{|c|c|c|}
\hline $\begin{array}{l}\text { Pobreza Contexto } \\
\text { Gobernanza débil } \\
\text { Capacidad técnica débil } \\
\text { Pocas opciones de medios de subsistencia } \\
\text { Bosques degradados }\end{array}$ & $\begin{array}{c}\text { Devolución de derechos/ formación de instituciones } \\
\text { forestales a nivel comunal } \\
\text { - Reconocimiento de los derechos de la comunidad a los bosques } \\
\text { - Concesión y registro del título o certificado } \\
\text { - Formación de instituciones comunitarias para recibir el título } \\
\text { - Demarcación de los límites de la comunidad }\end{array}$ & $\begin{array}{l}\text { Insumos e } \\
\text { intervención }\end{array}$ \\
\hline \multicolumn{3}{|c|}{$\begin{array}{l}\text { - Derechos no suficientemente amplios (por ejemplo, derechos para la comercialización de productos forestales } \\
\text { no maderables, pero no para madera) } \\
\text { - Comunidad escéptica sobre la inversión exterior } \\
\text { - Lapacidad comunal débil para gestionar alianzas comerciales } \\
\text { - Temor de que los valores tradicionales sean socavados con la exposición al mercado } \\
\text { - Tensión entre equidad social, beneficios medioambientales y maximización de ganancias }\end{array}$} \\
\hline \multicolumn{2}{|c|}{ Inversiones en desarrollo de capacidades de las instituciones forestales a nivel comunal } & $\begin{array}{l}\text { Resultados } \\
\text { intermedios }\end{array}$ \\
\hline \multirow{2}{*}{\multicolumn{2}{|c|}{$\begin{array}{l}\text { Cambios en las percepciones sobre los riesgos y garantías } \\
\text { - Tenencia percibida como segura } \\
\text { - Existen reglas que son entendidas ampliamente y aplicadas } \\
\text { - Mayor confianza en que los acuerdos se mantendrán } \\
\text { - Mayor capacidad para negociar de forma eficaz con actores externos }\end{array}$}} & Supuestos \\
\hline & & Resultados \\
\hline & \\
\hline $\begin{array}{r}\text { Rendimientos med } \\
\text { Mejores condiciones forestales, má } \\
\text { de la cc }\end{array}$ & $\begin{array}{l}\text { lioambientales, sociales y financieros positivos } \\
\text { is opciones de medios de subsistencia, mejoras en la infraestructura } \\
\text { omunidad, IFC viables financieramente }\end{array}$ & Impacto \\
\hline
\end{tabular}

Figura 1. Teoría del cambio que conecta la devolución de derechos con las inversiones financieras y los resultados medioambientales y sociales. 
- $\quad$ Premisa 3: La devolución de derechos a las comunidades ha promovido inversiones por parte de las IFC que generan resultados medioambientales y sociales, así como ganancias.

\section{Métodos}

\begin{tabular}{ll}
\hline Cuadro 1 - País y tipo de gestión de bienes comunes \\
\hline $\begin{array}{l}\text { Guatemala } \\
\text { México }\end{array}$ & $\begin{array}{l}\text { Concesiones forestales comunitarias (bosque) } \\
\text { Nepal }\end{array}$ \\
$\begin{array}{ll}\text { Grupos de usuarios de bosques comunitarios } \\
\text { (bosque) }\end{array}$ \\
Namibia & $\begin{array}{l}\text { Zonas de conservación de vida silvestre (vida } \\
\text { silvestre) }\end{array}$ \\
\hline
\end{tabular}

Usamos un enfoque comparativo de estudios de caso para evaluar los patrones en fuentes de inversión, mecanismos, sectores objetivo de inversión y rendimientos o beneficios esperados y obtenidos dentro de y en todos los países incluidos en el estudio. Guatemala, México y Nepal son países con procesos de devolución de derechos forestales a las comunidades, mientras que Namibia ha entregado los derechos sobre la vida silvestre. El tipo de gestión de bienes comunes en cada país está descrito en el Cuadro 1.

\section{Resultados}

\section{Resumen de la devolución de derechos}

En Guatemala, los contratos de concesión comunitaria, son acuerdos legales entre el Estado y un grupo organizado de personas que viven en comunidades locales, son un elemento clave de la devolución de los derechos forestales. Las disposiciones para implementar las concesiones forestales comunitarias fueron presentadas en 1994 y modificadas en 1998. Las negociaciones del Acuerdo de Paz de 1996 garantizaron los derechos de comunidades locales a los recursos en las áreas protegidas. Por medio de contratos de 25 años, los miembros de una concesión cuentan con derechos para manejar y extraer madera y productos forestales no maderables e implementar actividades de ecoturismo en áreas protegidas. El acceso de las comunidades y los derechos son otorgados bajo la condición de que las especies maderables de alto valor sean reguladas y certificadas (Radachowsky et al. 2012).

En México, la devolución a las comunidades de los derechos a la tierra comenzó con la revolución a inicios de la década de 1920 (Bray y Merino, 2002). Actualmente existen dos tipos de regímenes de tenencia comunal y formas de manejo de recursos de uso común — basados en las comunidades agrarias y ejidos. Los regímenes de manejo y tenencia de las comunidades indígenas se sustentan en el reconocimiento de los derechos consuetudinarios, mientras que los de los ejidos se basan en una forma de tenencia que surgió de las reformas agrarias. Aproximadamente la mitad de la superficie terrestre de México ha sido formalmente reconocida como ejidos o tierras comunitarias indígenas y un estimado de 9000 comunidades tienen bosques en sus tierras (INEGl, 1997). En 2017, más de
2134 ejidos y comunidades tenían permisos de manejo forestal (Carrillo-Anzures et al. 2017).

El manejo forestal en Nepal avanzó gradualmente hacia modalidades de base comunitaria que culminaron en la Ley Forestal de 1993, que legalizó las diferentes formas de manejo forestal de las comunidades y reconoció las IFC como organizaciones autónomas, perpetuas y corporativas que podían adquirir, poseer, transferir y administrar bienes muebles o inmuebles. En 2017, Nepal tenía 22266 bosques comunales que beneficiaba a 2,9 millones de hogares y cubrían un aproximado de 23,37 millones de hectáreas (Bhandari et al. 2019).

En 1996, Namibia institucionalizó el manejo comunitario de los recursos naturales (MCRN). Bajo esta forma de tenencia, los residentes de áreas comunales pueden formar una entidad de bienes comunes, llamada zona de conservación, para manejar, usar y recibir beneficios de la vida silvestre y otros recursos naturales en sus tierras tradicionales (Naidoo et al. 2016). Para registrar una zona de conservación, los habitantes deben desarrollar planes de manejo de los recursos, inscribirse para ser miembros y establecer un comité de gestión de la misma y una constitución. En 2017, Namibia había registrado 83 zonas de conservación que cubrían un estimado de 163000 km2 (MET/NACSO, 2018).

\section{Inversiones que suceden a la devolución de derechos}

El surgimiento de derechos locales más claros y sólidos sobre los recursos después de la devolución de derechos en cada uno de los cuatro casos por país catalizó las inversiones públicas y privadas resumidas en la Tabla 1.

\section{Análisis}

Premisa 1 - Las barreras a las inversiones en bienes comunes no son insuperables y se está produciendo inversión en recursos que son propiedad y están bajo el uso de las comunidades. La inversión externa proviene principalmente de donantes y gobiernos, pero la inversión del sector privado está aumentando.

- Los donantes, incluyendo los proveedores de asistencia oficial para el desarrollo (AOD) e instituciones financieras de desarrollo (IFD), parecen ser los inversionistas más importantes en los recursos de la comunidad.

- El apoyo del sector público nacional varía. La inversión gubernamental fue marcada en México, Namibia y Nepal, pero algo débil en Guatemala. En Namibia y Nepal, una porción grande de la inversión del gobierno se obtiene de las agencias de donantes.

- Existe inversión privada limitada en los cuatro casos, con garantías que se ofrecen en diferentes formas. Las inversiones conjuntas son comunes en Namibia y México. El arriendo simple o los acuerdos de compra/venta (off-take) con las comunidades se observan en Nepal y Namibia. La financiación mixta, en la que un banco de desarrollo o donante puede asumir parte del primer riesgo de pérdida en un préstamo, se está usando cada vez más en Nepal y Guatemala. 
Tabla 1. Inversiones posteriores a la devolución de derechos.

\begin{tabular}{|c|c|c|c|c|}
\hline & Guatemala & México & Nepal & Namibia \\
\hline Donantes & $\begin{array}{l}\text { Inversión financiera } \\
\text { importante que comenzó } \\
\text { en la década de } 1980 \\
\text { - } \quad \text { respaldo a } \\
\text { instituciones } \\
\text { públicas y desarrollo } \\
\text { y gobernanza de } \\
\text { concesiones } \\
\text { - asistencia técnica } \\
\text { - apoyo a instituciones } \\
\text { de segundo nivel }\end{array}$ & $\begin{array}{l}\text { Inversión moderada (a partir } \\
\text { de la década de 1980) que } \\
\text { involucra a entidades de } \\
\text { gobierno y paraestatales } \\
\text { - } \quad \text { creación/ } \\
\text { fortalecimiento de } \\
\text { instituciones; desarrollo } \\
\text { de capacidades de } \\
\text { gobernanza y gestión } \\
\text { asistencia técnica } \\
\text { asistencia con } \\
\text { certificaciones (por } \\
\text { medio de ONG) }\end{array}$ & $\begin{array}{l}\text { Inversión financiera importante } \\
\text { que data de la década de } 1980 \\
\text { - } \quad \text { capacitación técnica } \\
\text { desarrollo de capacidades } \\
\text { en gobernanza y manejo } \\
\text { forestal } \\
\text { desarrollo de infraestructura }\end{array}$ &  \\
\hline $\begin{array}{l}\text { Sector } \\
\text { público }\end{array}$ & $\begin{array}{l}\text { Inversión financiera menor; } \\
\text { la mayoría de fondos } \\
\text { estatales provienen de } \\
\text { donantes y bancos de } \\
\text { desarrollo } \\
\text { - gobernanza } \\
\text { forestal y fomento } \\
\text { de capacidades } \\
\text { de gestión de las } \\
\text { instituciones públicas } \\
\text { implementación de } \\
\text { políticas } \\
\text { aplicación de la ley }\end{array}$ & $\begin{array}{l}\text { Inversión financiera } \\
\text { significativa; cantidades } \\
\text { importantes desde los } \\
\text { donantes / bancos de } \\
\text { desarrollo } \\
\text { - } \quad \text { desarrollo de capacidades } \\
\text { para participar en alianzas } \\
\text { paraestatales (década de } \\
\text { 1960) } \\
\text { inversión paraestatal en } \\
\text { desarrollo institucional } \\
\text { (ejidos y uniones de } \\
\text { ejidos), infraestructura y } \\
\text { capital humano (década } \\
\text { de 1970) } \\
\text { desarrollo de EFC } \\
\text { (década de 1990) } \\
\text { manejo y conservación } \\
\text { (décadas de 1990/2000) }\end{array}$ & $\begin{array}{l}\text { Inversión financiera importante } \\
\text { pero la mayoría proveniente de } \\
\text { donantes/ bancos de desarrollo } \\
\text { - } \quad \text { capacitación técnica } \\
\text { - } \quad \text { desarrollo de capacidades } \\
\text { de gobernanza forestal y } \\
\text { - manejo forestal en IFC } \\
\text { - desarrollo de infraestructura }\end{array}$ & $\begin{array}{l}\text { Inversión financiera } \\
\text { importante pero la mayoría } \\
\text { proveniente de donantes/ } \\
\text { bancos de desarrollo } \\
\text { - } \quad \text { desarrollo de políticas } \\
\text { - mapeo y licenciamiento } \\
\text { de zonas de } \\
\text { conservación } \\
\text { concientización sobre } \\
\text { nuevas reglas } \\
\text { - capacitación } \\
\text { planificación a largo } \\
\text { plazo } \\
\text { dotación de personal y } \\
\text { vehículos }\end{array}$ \\
\hline $\begin{array}{l}\text { Instituciones } \\
\text { forestales } \\
\text { a nivel } \\
\text { comunal }\end{array}$ & $\begin{array}{l}\text { Inversión financiera } \\
\text { importante (relativa a } \\
\text { ingresos) a medida que los } \\
\text { concesionarios adquieren } \\
\text { competencias } \\
\text { - desarrollo de } \\
\text { capacidades para } \\
\text { gestionar /administrar } \\
\text { concesiones } \\
\text { planes de manejo } \\
\text { forestal } \\
\text { desarrollo de cadenas } \\
\text { de valor } \\
\text { fortalecimiento de } \\
\text { derechos } \\
\text { protección contra } \\
\text { incendios } \\
\text { empleos para los } \\
\text { miembros de la } \\
\text { concesión y otros } \\
\text { hogares } \\
\text { salud y educación } \\
\text { Instituciones de segundo } \\
\text { nivel -la Asociación de } \\
\text { Comunidades Forestales } \\
\text { de Petén (ACOFOP) y la } \\
\text { Empresa Comunitaria de } \\
\text { Servicios del Bosque Ltd. } \\
\text { (FORESCOM) son claves para } \\
\text { obtener fondos adicionales/ } \\
\text { expandir la influencia }\end{array}$ & $\begin{array}{l}\text { Inversiones financieras } \\
\text { importantes (relativas a } \\
\text { ingresos) a medida que las IFC } \\
\text { adquieren competencias } \\
\text { - } \quad \text { planes de manejo } \\
\text { forestal } \\
\text { desarrollo de cadenas } \\
\text { de valor /equipo e } \\
\text { instalaciones } \\
\text { mejora/ conservación } \\
\text { forestal } \\
\text { empleos para los } \\
\text { miembros de la } \\
\text { comunidad } \\
\text { infraestructura vial, } \\
\text { educación, salud } \\
\text { Numerosas instituciones de } \\
\text { segundo nivel (uniones de } \\
\text { ejidos, empresas conjuntas } \\
\text { Las asociaciones para } \\
\text { crear empresas conjuntas } \\
\text { entre comunidades hacen } \\
\text { inversiones financieras en } \\
\text { desarrollo de empresas y } \\
\text { bienes públicos }\end{array}$ & $\begin{array}{l}\text { Inversiones financieras } \\
\text { importantes (relativas a ingresos) } \\
\text { a medida que las IFC adquieren } \\
\text { competencias } \\
\text { - } \quad \text { empleos para los miembros } \\
\text { - } \quad \text { empresas forestales } \\
\text { - } \quad \text { planes de manejo forestal } \\
\text { - forestales } \\
\quad \text { caminos, agua, educación, } \\
\quad \text { atención médica } \\
\text { La Federación de Usuarios } \\
\text { Forestales Comunales de Nepal } \\
\text { (FECOFUN, por sus siglas en } \\
\text { inglés) desempeña una función } \\
\text { principal en el fortalecimiento de } \\
\text { los derechos comunales de uso } \\
\text { de los bosques y en el desarrollo } \\
\text { de capacidades de las IFC }\end{array}$ & $\begin{array}{l}\text { Inversiones financieras } \\
\text { importantes (relativas a } \\
\text { ingresos) a medida que las IFC } \\
\text { adquieren competencias } \\
\text { - } \quad \text { creación de empleos } \\
\text { - } \quad \text { empresas de ecoturismo } \\
\text { y } \\
\text { caza para conservación } \\
\text { - } \quad \text { infraestructura } \\
\text { - } \quad \text { educación/atención } \\
\quad \text { médica } \\
\text { planes de manejo de } \\
\quad \text { protección de la vida } \\
\text { Cilvestre } \\
\text { Carece de una asociación } \\
\text { nacional de zonas de } \\
\text { conservación; las asociaciones } \\
\text { regionales de zonas de } \\
\text { conservación son miembros } \\
\text { de la Asociación Namibiana } \\
\text { de Apoyo a las Organizaciones } \\
\text { CBNRM -Gestión de recursos } \\
\text { naturales basada en la } \\
\text { comunidad-(NACSO, por sus } \\
\text { siglas en inglés) }\end{array}$ \\
\hline
\end{tabular}




\begin{tabular}{|c|c|c|c|c|}
\hline & Guatemala & México & Nepal & Namibia \\
\hline $\begin{array}{l}\text { Sector } \\
\text { privado }\end{array}$ & $\begin{array}{l}\text { Inversión financiera } \\
\text { limitada hasta el momento, } \\
\text { pero están empezando } \\
\text { a surgir las asociaciones } \\
\text { entre bancos de desarrollo } \\
\text { y bancos comerciales para } \\
\text { proporcionar créditos a las } \\
\text { empresas forestales }\end{array}$ & $\begin{array}{l}\text { Compañía maderera } \\
\text { subsidiada con fondos } \\
\text { públicos-las asociaciones } \\
\text { comunitarias que } \\
\text { datan de la década } \\
\text { de } 1960 \text { invirtieron } \\
\text { en la capacitación de } \\
\text { las comunidades para } \\
\text { participar en asociaciones } \\
\text { Inversiones paraestatales } \\
\text { en infraestructura y } \\
\text { capital humano en la } \\
\text { década de } 1970 \text { sentaron } \\
\text { las bases para que surjan } \\
\text { las empresas forestales } \\
\text { comunitarias } \\
\text { Son comunes las alianzas } \\
\text { entre comunidades y } \\
\text { compradores }\end{array}$ & $\begin{array}{l}\text { Participación limitada del } \\
\text { sector privado externo } \\
\text { Las empresas forestales } \\
\text { pequeñas y medianas han } \\
\text { comenzado a invertir en } \\
\text { procesamiento de madera, } \\
\text { actividades turísticas, } \\
\text { procesamiento de productos } \\
\text { forestales no maderables y } \\
\text { comercialización } \\
\text { Ha surgido recientemente un } \\
\text { programa financiero mixto } \\
\text { que involucra a varios bancos } \\
\text { de desarrollo, al Gobierno de } \\
\text { Nepal y las comunidades }\end{array}$ & $\begin{array}{l}\text { - Participación sólida del } \\
\text { sector privado. Existen } \\
\text { numerosas empresas } \\
\text { conjuntas entre las } \\
\text { zonas de conservación } \\
\text { y operadores del sector } \\
\text { privado } \\
\text { Una fuente alternativa } \\
\text { emergente de ingresos } \\
\text { es un flujo de créditos e } \\
\text { incentivos para la vida } \\
\text { silvestre, que conecta el } \\
\text { desempeño de la zona } \\
\text { de conservación con la } \\
\text { inversión }\end{array}$ \\
\hline ONG & $\begin{array}{l}\text { Los donantes usualmente } \\
\text { canalizan su ayuda a las IFC } \\
\text { a través de las ONG } \\
\text { - } \quad \text { habilidades de } \\
\text { manejo forestal } \\
\text { - } \quad \begin{array}{l}\text { planes de manejo } \\
\text { forestal }\end{array} \\
\text { certificación del } \\
\text { Consejo de Manejo } \\
\text { Forestal (FSC, por sus } \\
\text { siglas en inglés) } \\
\text { capacitación en } \\
\text { gestión empresarial, } \\
\text { desarrollo de cadenas } \\
\text { de valor (productos } \\
\text { forestales maderables } \\
\text { y no maderables) } \\
\text { promoción de } \\
\text { reformas políticas }\end{array}$ & $\begin{array}{l}\text { Los donantes usualmente } \\
\text { canalizan su ayuda a las IFC a } \\
\text { través de las ONG } \\
\text { - } \quad \begin{array}{l}\text { planes de manejo } \\
\text { forestal }\end{array} \\
\text { - } \quad \text { habilidades de manejo } \\
\text { forestal } \\
\text { certificación del Consejo } \\
\text { de Manejo Forestal (FSC, } \\
\text { por sus siglas en inglés) } \\
\text { capacitación en gestión } \\
\text { - empresarial } \\
\text { desarrollo de cadenas } \\
\text { de valor } \\
\text { promoción de reformas } \\
\text { políticas }\end{array}$ & $\begin{array}{l}\text { Los donantes generalmente } \\
\text { canalizan su ayuda a las IFC a } \\
\text { través de las ONG } \\
\text { - } \quad \text { planes de manejo forestal } \\
\text { - } \quad \text { habilidades para el manejo } \\
\text { forestal } \\
\text { certificación del Consejo de } \\
\text { Manejo Forestal (FSC, por } \\
\text { sus siglas en inglés) para } \\
\text { productos forestales no } \\
\text { maderables } \\
\text { capacitación en habilidades } \\
\text { financieras / administración } \\
\text { de negocios } \\
\text { desarrollo de cadenas } \\
\text { de valor para productos } \\
\text { forestales no maderables } \\
\text { promoción de reformas } \\
\text { políticas }\end{array}$ & $\begin{array}{l}\text { Un consorcio de ONG locales } \\
\text { e internacionales fundó la } \\
\text { Asociación Namibiana de } \\
\text { Apoyo a las Organizaciones } \\
\text { CBNRM -Gestión de recursos } \\
\text { naturales basada en la } \\
\text { comunidad- (NACSO, por sus } \\
\text { siglas en inglés) } \\
\text { - } \quad \text { asistencia legal para el } \\
\text { registro de zonas de } \\
\text { conservación y acuerdos } \\
\text { de inversión conjunta } \\
\text { monitoreo de la vida } \\
\text { silvestre / } \\
\text { sistemas de seguros por } \\
\text { daños a la vida silvestre } \\
\text { capacitación técnica } \\
\text { financiera y administrativa } \\
\text { desarrollo de cadenas de } \\
\text { valor de los productos del } \\
\text { veld (paisaje rural abierto) }\end{array}$ \\
\hline
\end{tabular}

Premisa 2 - La preparación para la inversión requiere que los inversionistas potenciales y quienes reciben la inversión tengan una garantía de que las obligaciones de cada parte serán cumplidas. Cada sector asume funciones y responsabilidades específicas para mitigar los riesgos. $L a$ preparación para las inversiones se desarrolla con el tiempo y en etapas a medida que los niveles de garantía se incrementan.

- Los donantes y las inversiones gubernamentales fueron esenciales para el desarrollo de capacidades de las IFC al inicio en áreas como gobernanza, educación financiera, administración, elaboración de planes de negocios y manejo forestal.

- Con frecuencia, los donantes canalizan los fondos a través de ONG o consultores para desarrollar capacidades, negociar tratos entre inversionistas y comunidades, así como para dar garantías a los inversionistas en cuanto a que tendrán los retornos esperados.

- La formación de nuevas alianzas contribuyó al surgimiento de IFC financieramente viables. El apoyo de las ONG permitió que las IFC obtuvieran la certificación del Consejo de Manejo Forestal (FSC, por sus siglas en inglés) para madera en Guatemala y México y para productos forestales no maderables en Nepal. En Namibia, los socios del sector privado proporcionaron capacitación que permitió que las zonas de conservación lograran estándares internacionales en hotelería y turismo.

- Las asociaciones a nivel nacional han desempeñado un rol clave para que surjan IFC viables financieramente.

Por medio de organizaciones de segundo nivel, las IFC han promovido exitosamente reformas de políticas que han mejorado las condiciones habilitantes para las empresas comunales.

- Se están experimentando nuevas prácticas en cuanto a mecanismos financieros que luego son institucionalizadas.

- La Asociación Namibiana de Apoyo a las

Organizaciones CBNRM -Gestión de recursos naturales basada en la comunidad- (NACSO, por sus siglas en inglés), ha creado un fondo para la conservación para atraer fondos de diversas fuentes y canalizarlas a las zonas de conservación.

- En México, algunas IFC han desarrollado asociaciones intercomunitarias innovadoras que les permiten obtener economías de escala. 
- Los concesionarios forestales en Guatemala están experimentando con mecanismos de financiamiento mixto que incluyen una combinación de financiamiento multilateral y crédito bancario.

- En Nepal, algunas IFC han diseñado mecanismos de autofinanciamiento como cuotas de membresía estratificadas para cubrir los costos de capacitación y equipos.

Nuestros resultados respaldan la propuesta de Kluvánková et al. (2018) en cuanto a que las empresas forestales comunitarias son parte de un sistema dinámico de innovación social más amplio. Este sistema es generado por la devolución de derechos a las comunidades. Las inversiones se materializan en etapas, con niveles de garantía y necesidades que cambian a medida que el sistema evoluciona (Figura 2). La Figura 3 describe nuestro diagrama TC revisado que refleja la naturaleza de las fases del sistema de innovación social.

Premisa 3 - Los derechos de las comunidades han promovido inversiones que reconocen el carácter social de la propiedad y el uso de bienes comunes que producen resultados medioambientales y sociales, así como ganancias.

- Las IFC enfatizaron la creación de oportunidades de empleo incluso a costa de la pérdida de algo de su competitividad financiera. Dieron preferentemente empleos de medio tiempo a todos los miembros de la comunidad que quisieran trabajar, en vez de ofrecer empleo de tiempo completo a unos pocos seleccionados.

\section{- Las IFC han asumido algunas funciones} gubernamentales, específicamente en la provisión de bienes públicos como caminos, escuelas y centros de salud.

- Las IFC comúnmente hacen inversiones con el objetivo de mejorar la productividad del bosque (o de la vida silvestre), las que tienden a producir resultados medioambientales positivos.

\section{Conclusiones}

Señalamos tres características claves tomadas de los casos que pueden orientar las políticas y programas que buscan respaldar las inversiones en IFC.

- Fuentes diferentes de inversión financiera intervienen en diferentes etapas. Las inversiones del sector público y de donantes son críticas en la primera etapa, cuando los riesgos para los inversionistas son más altos. Estas continúan en la etapa dos, cuando los riesgos y costos de transacción, a pesar de ser menores, continúan siendo altos. La inversión financiera del sector privado se incrementa en la etapa tres, una vez que los riesgos y costos de transacción se reducen.

- Los tipos de inversión necesarios cambian a medida que el sistema de innovación social ingresa en una nueva etapa. Las inversiones habilitantes se requieren inicialmente para establecer la infraestructura social necesaria para que las comunidades actualicen sus derechos. Durante la segunda etapa, estas inversiones continúan siendo importantes pero las inversiones en activos ganan relevancia a medida que aumentan la posibilidad de que las IFC ofrezcan un suministro adecuado de productos con las cualidades deseadas. En la etapa tres, las inversiones en activos empiezan a ser dominantes.

- La evolución de las inversiones en las IFC es repetitiva. Los resultados de las inversiones habilitantes iniciales alteran el contexto y catalizan la necesidad de inversiones en activos, así como de inversiones habilitantes adicionales. Por medio de un proceso constante de aprendizaje y adaptación, las IFC fomentan la confianza de sus miembros y de los inversionistas externos en relación con su capacidad para ofrecer retornos financieros adecuados.

Las comunidades, gobiernos y el sector privado tienen que ser vistos por todas las partes como socios del mismo valor en los sistemas de innovación social que crean y mantienen las condiciones que permiten la existencia de las empresas comunitarias con visión social y sirven para el logro de una variedad de objetivos medioambientales, sociales y económicos. Un entendimiento común del carácter sistémico de los procesos de innovación no puede siempre darse por sentado, y algunas partes, especialmente en el gobierno y el sector privado, no comprenden que también son necesarios los cambios fundamentales y permanentes en algunas de sus propias políticas y prácticas. Las agencias forestales necesitan revisar sus funciones enfocadas en esquemas restrictivos del uso forestal e invertir más en la protección de los derechos de las comunidades y el desarrollo de capacidades de las organizaciones locales.

Las empresas privadas necesitan tener la voluntad para incorporar los objetivos de bienestar social y empleo de las IFC. Se necesita investigación adicional sobre las fuentes, los mecanismos, el volumen y el rumbo de las inversiones en recursos manejados por las comunidades que puedan crear el ámbito de acción para la innovación social. Debido a las necesidades cambiantes de capital de las IFC a medida que crecen, sostenemos que es importante centrar la investigación en lograr una comprensión mejor de sus opciones financieras y potencial de inversión en las diferentes etapas de su desarrollo.

\section{Agradecimientos}

El financiamiento del Programa de Investigación del CGIAR sobre Políticas, Instituciones y Mercados (PIM) conducido por el Instituto Internacional de Investigación sobre Políticas Alimentarias (IFPRI, por sus siglas en inglés) y el Centro para la Investigación Forestal Internacional (CIFOR) respaldó este trabajo.

Este infobrief fue elaborado a partir de una ponencia presentada en la Conferencia Tierra y Pobreza del Banco Mundial en 2018: Gobernanza de la tierra en un mundo interconectado. Washington D.C. 19-23 marzo de 2018. 07-07: Derechos comunales para beneficio del medio ambiente por Lawry S, Gynch S, Monterroso I y Adhikary A. 2018. Beneficios de los bienes comunes: cómo la tenencia comunitaria está facilitando la inversión en los bienes comunes para el crecimiento inclusivo. 
Etapa 1 - Inversión en la devolución de derechos e instituciones de gobernanza

- La etapa uno se caracteriza por las inversiones para realizar la devolución de derechos y facilitar el surgimiento de IFC que puedan gobernar los bosques $u$ otros recursos de propiedad o uso común eficazmente.

- El Estado y los donantes son los inversionistas externos dominantes durante esta etapa, con ONG locales e internacionales que sirven principalmente como intermediarias entre las IFC y las agencias estatales, así como entre las IFC y los donantes.

- Las barreras para las inversiones en empresas asociadas con las IFC normalmente existen y deben ser superadas.

- Durante esta etapa, las inversiones encajan en la categoría de "inversiones habilitantes" de Elson (2010).

Figura 2. Etapas de inversión en IFC.
Etapa 2 - Inversión en gobernanza y capacidades técnicas

- La atención se centra en el desarrollo de capacidades administrativas y de gestión organizacional en las IFC. Esto, en conjunto con el desarrollo de capacidades técnicas y las actividades de gestión asociadas, mejora la base de recursos naturales.

- Inversiones adicionales respaldan a las empresas comunitarias en mercados establecidos como madera y turismo.

- Los donantes y el Estado continúan siendo los inversionistas externos dominantes y las ONG siguen funcionando como intermediarias.

- Las IFC, frecuentemente con el apoyo de ONG, establecen federaciones que representan sus intereses, promueven reformas de políticas y las ayudan a lograr economías de escala.

- Las inversiones encajan primariamente en la categoría de inversiones habilitantes, pero se hacen algunas inversiones en activos.
Etapa 3 - Inversión en

empresas

- Las reducciones en los costos de transacción brindan las garantías que atraen a un conjunto más amplio de inversionistas del sector privado.

- Las federaciones de IFC se enfocan inicialmente en negociar un contexto regulatorio de respaldo. Una vez que las condiciones son más favorables, ellas ponen más atención en la promoción de inversiones comerciales.

- El uso forestal certificado y los planes de extracción contribuyen a que las IFC cumplan las condiciones estrictas de los compradores internacionales y les permiten atraer inversionistas que demandan estándares altos.

- Las empresas de las IFC cimentan su carácter social, aseguran que los sectores pobres de la comunidad reciban apoyo y que una parte de los ingresos excedentes se invierta en la provisión de bienes públicos.

- Los donantes y el Estado continúan haciendo inversiones en las empresas de las IFC en esta etapa, pero las inversiones del sector privado externo comienzan a expandirse.

- El énfasis se desplaza a las inversiones en activos, aunque se siguen haciendo inversiones habilitantes, tales como la adquisición de la certificación FSC.

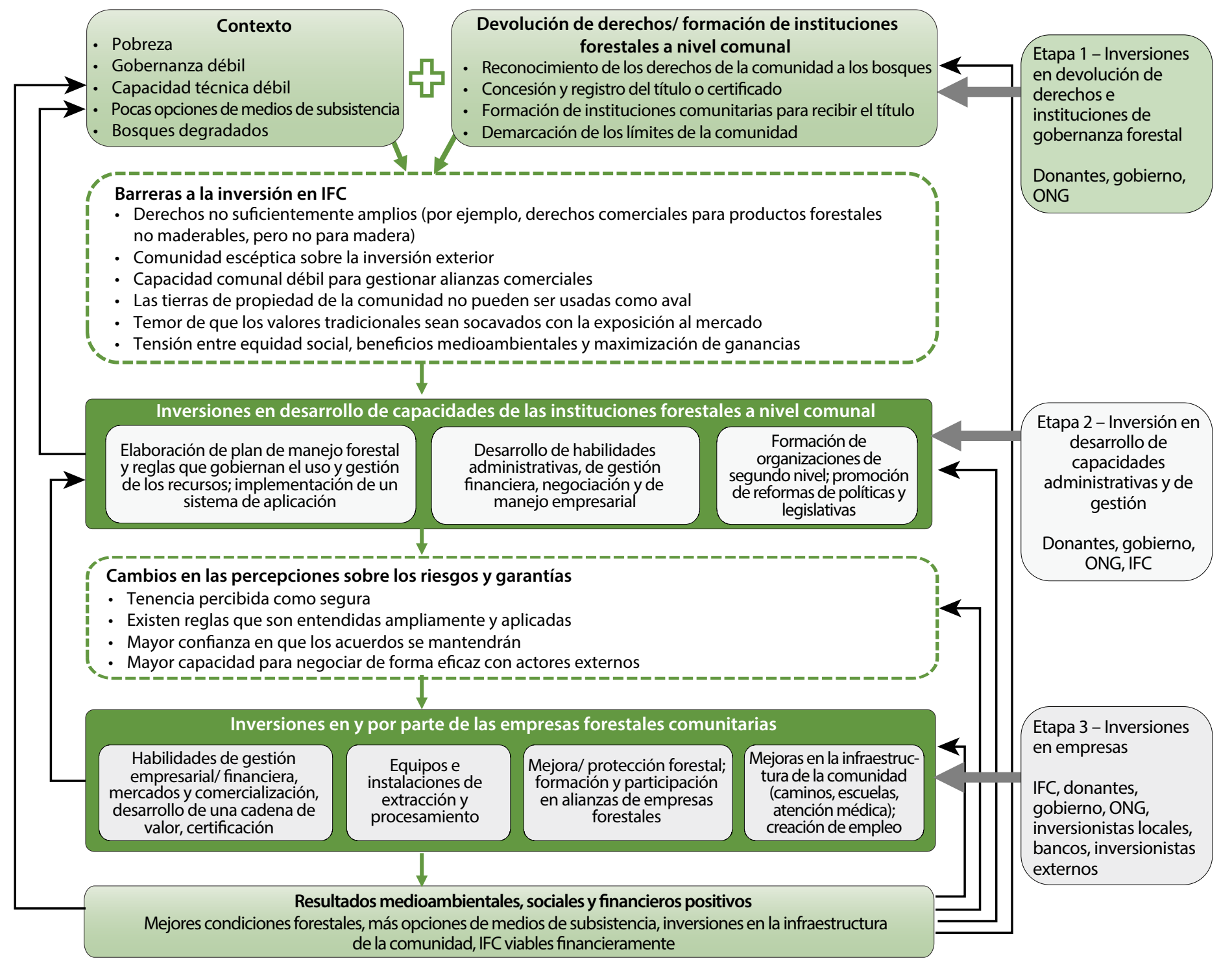

Figura 3. Teoría de cambio adaptada que conecta la devolución de derechos, inversiones y resultados. 


\section{Referencias}

Ambrose-Oji B, Lawrence A y Stewart A. 2015. Community based forest enterprises in Britain: two organizing typologies. Forest Policy and Economics 58:65-74.

Baynes J, Herbohn J, Smith C, Fisher R y Braye D. 2015. Key factors which influence the success of community forestry in developing countries. Global Environmental Change 35:226-238.

Bhandari PKC, Bhusal P, Paudel G, Upadhyaya CP y Chhetri BBK. 2019 Importance of community forestry funds for rural development in Nepal. Resources 8(2):85. doi:10.3390/resources8020085

Bray D y Merino L. 2002. The rise of community forestry in Mexico: History, concepts, and lessons learned from twenty years of community timber production (p. 133). Project Report. México, D.F.: Ford Foundation.

Carrillo-Anzures M, Flores E, Torres Rojo J, Sangerman-Jarquín D, Gonzalez L y Buendía E. 2017. Characterization of forest producers in 12 states of the Mexican Republic. Revista Mexicana de Ciencias Agrícolas 8(7):1561-73.

Cronkleton P, Bray DB y Medina G. 2011. Community forest management and the emergence of multi-scale governance institutions: lessons for REDD+ development from Mexico, Brazil and Bolivia. Forests 2:451-73.

Dasgupta P. 2005. Common property resources: Economic analytics. Economic and Political Weekly 40(16):1610-1622.

Elson D. 2012. Guide to investing in locally controlled forestry. Growing forest partnerships in association with FAO, IIED, IUCN, The Forests Dialogue and the World Bank. Londres, Reino Unido: International Institute for Environment and Development

Fellnhofer K. 2015. Literature review: investment readiness level of small and medium sized companies. International Journal of Managerial and Financial Accounting 7(3/4):268-84.

Foundjem-Tita D, Duguma LA, Speelman S y Piabuo SM. 2018. Viability of community forests as social enterprises: a Cameroon case study. Ecology and Society 23(4):50. doi.org/10.5751/ ES-10651-230450

Hewitt D y Castro Delgadillo M. 2009. Key factors for successful community-corporate partnerships - results of a comparative analysis among Latin American cases. Richmond, Vermont, EEUU: Rainforest Alliance.

Kluvánková T, Brnkalákova S, Špaček M, Slee B, Nijnik M, Valero D, Miller D, Bryce R, Kozova M, Polman N, Szabo Ty Gežík V. 2018. Understanding social innovation for the wellbeing of forestdependent communities: A preliminary theoretical framework. Forest Policy and Economics 97:163-74.

Lawry S, Samii C, Hall R, Leopold A, Hornby D y Mtero F. 2017. The impact of land property rights interventions on investment and agricultural productivity in developing countries: a systematic review. Journal of Development Effectiveness 9(1):61-81.

Mason C y Kwok J. 2010. Investment readiness programmes and access to finance: a critical review of design issues. Local Economy 25(4):269-92.

[MET/NACSO] Ministry of Environment and Tourism/Namibian Association of CBNRM Support Organisations. 2018. The state of community conservation in Namibia - a review of communal conservancies, community forests and other CBNRM activities (Annual Report 2017). Windhoek, Namibia: MET/NACSO.

Naidoo R, Weaver C, Diggle RW, Greenwell M, Stuart-Hill G and Thouless C. 2016. Complementary benefits of tourism and hunting to communal conservancies in Namibia. Conservation Biology 30(3):628-38.

Paudel NS, Monterroso I and Cronkleton P. 2012. Secondary level organisations and the democratisation of forest governance: Case studies from Nepal and Guatemala. Conservation and Society 10(2):124-135.

Polman N, Slee W, Kluvánková T, Dijkshoorn M, Nijnik M, Gezik V and Soma K. 2017. Classification of Social Innovations for Marginalized Rural Areas, Deliverable 2.1, Social Innovation in Marginalised Rural Areas (SIMRA). Brussels, Belgium: European Union Framework Programme Horizon 202. pp. 32.

Radachowsky J, Ramos VH, McNab R, Baur EH and Kazakov N. 2012. Forest concessions in the Maya Biosphere Reserve, Guatemala: A decade later. Forest Ecology and Management 268:18-28.

Wily LA. 2018. Collective land ownership in the 21st century: Overview of global trends. Land 7(2):68, https://doi.org/10.3390/ land7020068

Contacto: Steven Lawry (s.lawry@cgiar.org)


desarrollando las capacidades de sus socios y dialogando activamente con todos los actores involucrados, para informar sobre las políticas y las prácticas que afectan a los bosques y a las personas. CIFOR es un centro de investigación CGIAR y lidera su Programa de Investigación sobre Bosques, Árboles y Agroforestería (FTA por sus siglas en inglés). Nuestra sede central se encuentra en Bogor, Indonesia, y contamos con oficinas en Nairobi, Kenia; Yaundé, Camerún; Lima, Perú, y Bonn, Alemania. 